\title{
Impact of the Sternotomy Wires and Aortic Valve Implant on the On-Body UWB Radio Channels
}

\author{
Mariella Särestöniemi, Timo Kumpuniemi, \\ Matti Hämäläinen, Jari Iinatti \\ Centre for Wireless Communications (CWC) \\ University of Oulu \\ Oulu, Finland \\ givenname.familyname@oulu.fi
}

\author{
Carlos Pomalaza-Raéz \\ Department of Electrical and Computer Engineering \\ Purdue University \\ Fort Wayne, Indiana, USA \\ cpomalaz@purdue.edu
}

\begin{abstract}
In this paper, the impact of the sternotomy wires and aortic valve implant on the ultra wideband (UWB) channel characteristics is studied. The evaluations are performed by calculations, measurement data analysis, and power flow simulations. The aim is to show that implants, which consist of steel, titanium, and other highly conductive materials, do have clear effect on the signal propagation even inside the tissues. This impact should be taken into account when using in-body or onbody communications devices, such as capsule endoscopes, etc.
\end{abstract}

Keywords-aortic valve implant; radio channel; signal propagation; sternotomy closure wires; WBAN

\section{INTRODUCTION}

Wireless body area network (WBAN) channel characteristics have been under an intensive study during the recent years. Several channel models, measurement campaigns and propagation simulations have been realized to get deeper understanding of the channel propagation in the vicinity or even inside a human body [1-10].

Medical implants, such as pace makers, valve implants, etc. bring their own challenge on this field. Many of the implants may contain steel, titanium alloy, tungsten, and other highly conductive materials and thus will have clear effect on the channel characteristics in the vicinity where they are located. Furthermore, medical wires, staples, and bands, which are used for the closure after the operation [11], can have significant impact since usually they are located close to the skin and hence close to the on-body antennas and sensor nodes.

There are just a few studies on the impact of the medical implants on the channel characteristics [6-8]. The main challenge in this type of studies is the difficulty to obtain measurement data. Although the WBAN channel characteristics can be investigated through electromagnetic propagation simulations, which are based on, for instance, Finite Difference Time Domain (FDTD), Finite Integration Technique (FIT) or Finite Element Method (FEM) [9,10], at some phase the results should be verified with the real measurement data.

The impact of a titanium based aortic valve implant on the ultra wideband (UWB) channel characteristics has been reported in [6-8]. The studies in [6-7] included measurement data where one of the volunteers has an aortic valve implant. The main result of the previous work is that the titanium based aortic valve has an impact on the channel characteristics depending on the locations of the on-body antennas. Reference [8] applies this scenario in the FEM-based 3D simulator studying the impact of the aortic valve on different locations of the antennas as well as the impact of the depth of the valve.

To our best knowledge, there are no published studies on the impact of the post-surgery medical wires on the UWB on-body channel propagation. However, it is essential to understand their impact on the channel characteristics since these kinds of impacts have to be taken into account when using any in-body or on-body device, for instance endoscopy capsules or wearables.

The main contribution of this paper is to show the impact of the sternum closure wires on the propagation of UWB signals. The study is performed by calculations, measurement data analysis and FIT-based power flow simulations. Three different on-body antennas using two different separation distances are considered. In this study, the impact of the titanium based aortic valve on the channel characteristics has also been reported.

This paper is organized as follows: Section II presents the setups used in the simulations and measurement campaign. Section III presents the results of the power flow simulations. Calculations for propagation times are presented in Section IV. Measurement results are presented and discussed in Section V. Summary and Conclusions are given in Section VI.

\section{SIMULATION AND MEASUREMENT SETUP}

\section{A. Antennas}

In this study, we used three different antennas designed for on-body communication: loop and dipole antennas designed at CWC [12], and commercial SkyCross antennas [13]. The antennas were located on the chest asymmetrically respect to the sternum: i.e., one antenna was on the middle of the sternum and the other on the left side of the chest. Two antenna separation distance cases were studied: $6.5 \mathrm{~cm}$ and $15 \mathrm{~cm}$. Figure 1 illustrates the location of the antennas respect to the sternum, heart and the sternotomy wires. Figure 2 presents the location of the aortic valve in the heart. For an antenna separation distance $6.5 \mathrm{~cm}$, we studied also the case when the antenna is on the right 
side of the chest. Between the antenna and the human body, there was a $4 \mathrm{~mm}$ rohacell piece to improve antenna efficiency.

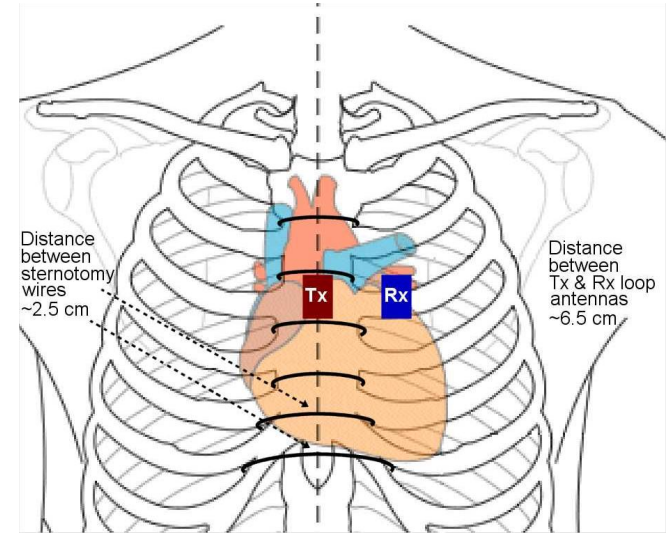

Figure 1. Location of the antennas on the chest for left side case.

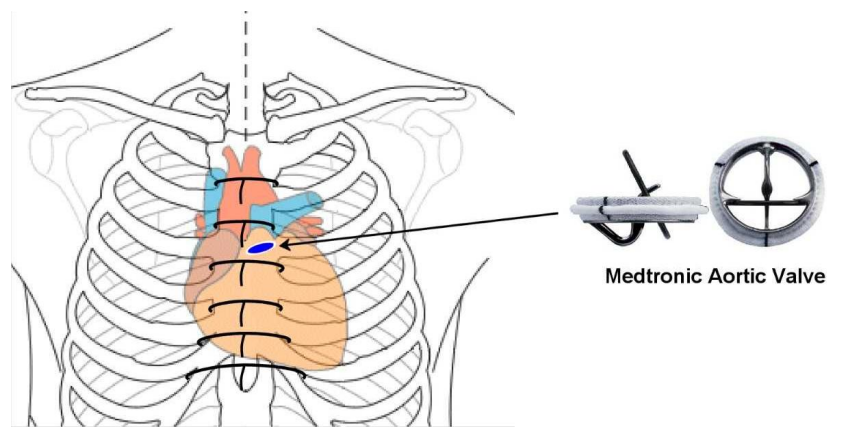

Figure 2. Location of the aortic valve in the heart.

\section{B. Simulator}

We studied the propagation inside the human body using the CST MicroWaveStudio (MWS) [14] simulation software with an extension of Biomodel library. An anatomical voxel model Tom was chosen for our simulations. A simplified version of an aortic valve implant was modelled according to the figures and information provided by Medtronics in [15], and included it in the heart of the voxel model. The picture of a Medtronics' valve is found in Figure 2 [15]. The aim of the simulations was to model the power flow inside the human body to find the possible reflections and diffractions from the sternotomy wires and the aortic valve.

\section{Measurements}

The measurements were conducted in an anechoic chamber. Two sets of measurements were taken. In the first measurement set, the frequency bandwidth was $2-8 \mathrm{GHz}$ and the antenna distance was $d_{\mathrm{A}}=6.5 \mathrm{~cm}$. Volunteers A (with implant) and $\mathrm{B}$ (without implant) participated into this measurement. In the second measurement set, in which the volunteers $\mathrm{A}$ and $\mathrm{C}$ (without implant) participated, the frequency bandwidth was 3.1-10.6 GHz and $d_{\mathrm{A}}=15 \mathrm{~cm}$. In both measurements, the number of frequency points per sweep was set to 1601 . The measurements were conducted in a frequency domain to obtain channel's frequency responses (S21 parameters,) which were later transformed into time domain in Matlab using Inverse Fast Fourier Transform (IFFT) to get impulse response (IR). Since the channel data is stored in the frequency domain, it will be possible to study impulse responses for different bandwidths as the IFFT can be performed to the selected bandwidth.

\section{Volunteers}

Three male volunteers assisted in the measurements. The sizes of the volunteers are summarizes in Table I. One of them has a titanium alloy based aortic valve implant (illustrated in Figure 2.) This subject also has medical wires in his sternum used in the sternum closure after the valve operations. The medical wires are made from steel and there are altogether six wires as shown in Figure 1.

\section{POWER FLOW Simulations}

First, the propagation inside the human body with an aortic valve implant was studied by simulating the power flow around the chest area. For this first scenario, we did not include yet the metallic wires in the model since we preferred to see the pure impact of the aortic valve. For the comparison, the propagation flow was simulated for the voxel model without the implant as well.

Figures $3 \mathrm{a}$ ja $3 \mathrm{~b}$ show the power flows with and without implant, respectively. We can observe that the implant has a clear effect on the propagation flow: the power flow is different in terms of spreading and intensity if the valve and wires are included in the model since there are several reflections from the titanium alloy torus ring, as well as from the steel wires.

Table I Information about the volunteers.

\begin{tabular}{|l|l|l|l|}
\hline & A(with implant) & B (no implant) & C (no implant) \\
\hline Height & $170 \mathrm{~cm}$ & $181 \mathrm{~cm}$ & $173 \mathrm{~cm}$ \\
\hline Weight & $62 \mathrm{~kg}$ & $76 \mathrm{~kg}$ & $75 \mathrm{~kg}$ \\
\hline Age & 61 & 22 & 27 \\
\hline
\end{tabular}

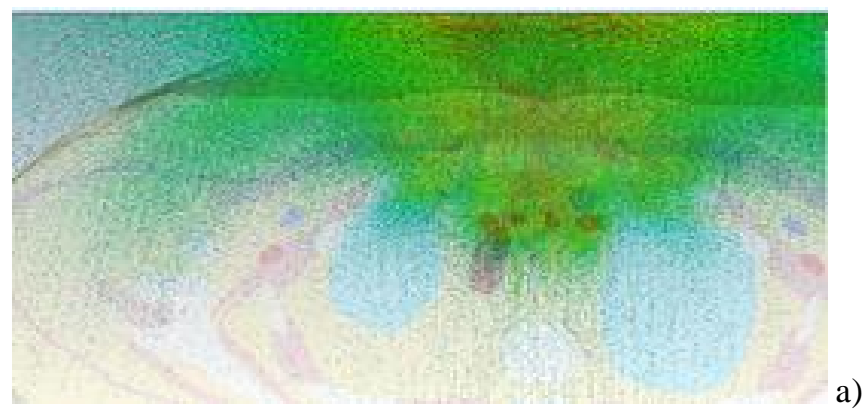

a)

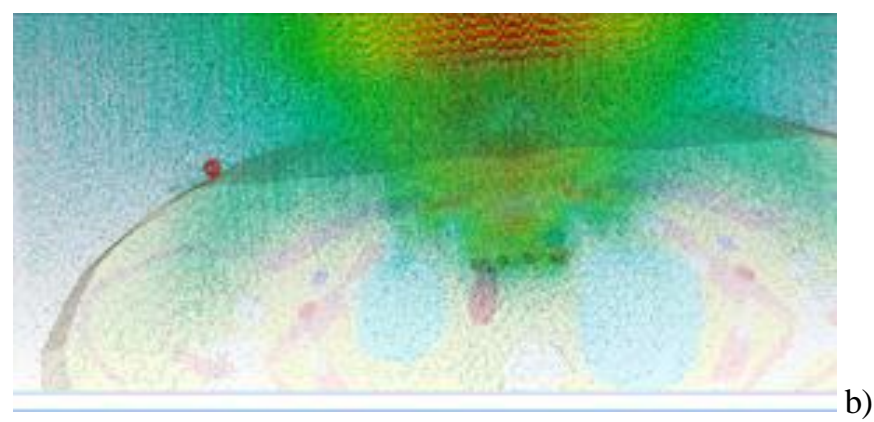

Figure $3 \mathrm{a}$ and $3 \mathrm{~b}$. Simulated power flow for the voxel model a) with a valve implant and b) without a valve implant. 


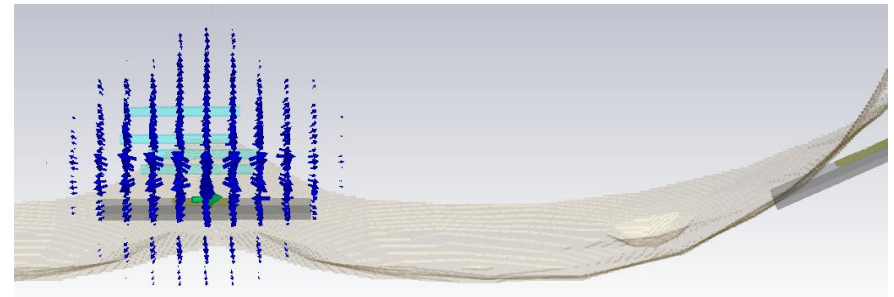

Figures 4. Reflections from the sternotomy wires.

Furthermore, the power flow study is repeated for the model with metallic wires used for sternum closure (Figure 4.) In this case, the valve implant is not included in the model. From the propagation flow figure, one can note reflections from metal wires towards RX-antenna.

\section{PROPAGATION TIME CALCULATIONS}

According to the propagation flows, there are several reflected propagation paths from the valve implant and sternotomy wires. Different paths go through different tissues and with different propagation times. Just some of the reflected signals arrive with power level high enough to be recognized in the RX-antenna. In this section, we calculate propagation time for signals reflecting from the valve and the sternotomy wires.

When calculating the reflection from the wire, we assume straightest reflection path, which is assumed to be the strongest one, from the wire towards RX-antenna. Basically, this signal would go through muscle, fat and skin tissues.

When we calculate the impact of the aortic valve on the channel characteristics, we also assume reflection from the valve or the surrounding torus ring towards RX. This signal would travel through all the tissues: heart, lung, bone, muscle, fat and skin. Next, we will calculate the assumed arrival time for the peaks due to the wires and the peaks due to the valve. The velocity of the propagation signal in the $i^{\text {th }}$ tissue is calculated as

$$
v_{i, f}=\frac{c}{\sqrt{\varepsilon_{i, f}}},
$$

where $\varepsilon_{i, f}$ is the relative permittivity of a tissue at frequency $f$. The relative permittivity may change significantly with the frequency depending on the tissue. Once we know the velocity, we can easily calculate the propagation time in each of the tissues having a length of $d_{\mathrm{i}}$ using

$$
t_{i, f}=\frac{d_{i}}{v_{i, f}},
$$

which are then summed up to obtain overall propagation time.

We made our assumptions of the $d_{i}$ based on the dimensions of the voxel model and information obtained from the anatomy references [11]. Parameter $d$ consist of the signal path going directly from TX-antenna to the valve $d_{\mathrm{TX}-\mathrm{V}}$ and then the reflected path from valve to $\mathrm{RX} d_{\mathrm{V}-\mathrm{RX}}$. In general, these distances are just assumptions since human bodies have different dimensions.
Table II. Relative permittivity $\left(\varepsilon_{r}\right)$ and depth of different tissues

\begin{tabular}{|c|c|c|c|c|c|c|}
\hline & Skin & Bone & Fat & Muscle & Heart & $\begin{array}{l}\text { Lung } \\
\text { (d/i) }\end{array}$ \\
\hline$\varepsilon_{\mathrm{r},}, 2 \mathrm{GHz}$ & 38.6 & 11.7 & 11.0 & 53.3 & 55.8 & $21 / 49$ \\
\hline$\varepsilon_{r}, 3 \mathrm{GHz}$ & 31.3 & 11.0 & 10.7 & 52.1 & 53.7 & $20 / 47$ \\
\hline$\varepsilon_{r}, 8 \mathrm{GHz}$ & 33.2 & 8.8 & 9.3 & 45.5 & 45.4 & $17 / 41$ \\
\hline$\varepsilon_{r}, 10 \mathrm{GHz}$ & 37.5 & 8.12 & 8.8 & 42.8 & 42.2 & $16 / 38$ \\
\hline $\begin{array}{l}d_{\mathrm{TX}-\mathrm{w}} \text { as } \\
d_{\mathrm{TX}, \mathrm{RX}}= \\
6.5 \mathrm{~cm}, \\
15 \mathrm{~cm}\end{array}$ & $0.005 \mathrm{~m}$ & - & $\begin{array}{l}0.005 \\
\mathrm{~m}\end{array}$ & - & - & - \\
\hline $\begin{array}{l}d_{\mathrm{w}-\mathrm{RX}, \mathrm{as}} \\
d_{\mathrm{TX}, \mathrm{RX}}= \\
6.5 \mathrm{~cm}\end{array}$ & $0.005 \mathrm{~m}$ & - & $\begin{array}{l}0.01 \\
\mathrm{~m}\end{array}$ & $\begin{array}{l}0.045 \\
\mathrm{~m}\end{array}$ & - & - \\
\hline $\begin{array}{l}d_{\mathrm{TX}-\mathrm{v}} \text { as } \\
d_{\mathrm{TX}, \mathrm{RX}}= \\
6.5 \mathrm{~cm}, \\
15 \mathrm{~cm}\end{array}$ & $0.005 \mathrm{~m}$ & $\begin{array}{l}0.01 \\
\mathrm{~m}\end{array}$ & $\begin{array}{l}0.005 \\
\mathrm{~m}\end{array}$ & $0.01 \mathrm{~m}$ & $\begin{array}{l}0.03 \\
\mathrm{~m}\end{array}$ & - \\
\hline $\begin{array}{l}d_{\mathrm{V}-\mathrm{RX}} \text { as } \\
d_{\mathrm{TX}, \mathrm{RX}}= \\
6.5 \mathrm{~cm}\end{array}$ & $0.005 \mathrm{~m}$ & $\begin{array}{l}0.01 \\
\mathrm{~m}\end{array}$ & $\begin{array}{l}0.005 \\
\mathrm{~m}\end{array}$ & $\begin{array}{l}0.001 \\
\mathrm{~m}\end{array}$ & $\begin{array}{l}0.02 \\
\mathrm{~m}\end{array}$ & $\begin{array}{l}0.025 \\
\mathrm{~m}\end{array}$ \\
\hline $\begin{array}{l}d_{\mathrm{w}-\mathrm{RX}, \mathrm{as}} \\
d_{\mathrm{TX}, \mathrm{RX}}= \\
15 \mathrm{~cm}\end{array}$ & $0.005 \mathrm{~m}$ & - & $\begin{array}{l}0.05 \\
\mathrm{~m}\end{array}$ & $\begin{array}{l}0.085 \\
\mathrm{~m}\end{array}$ & - & - \\
\hline $\begin{array}{l}d_{\mathrm{V}-\mathrm{RX}} \text { as } \\
d_{\mathrm{TX}, \mathrm{RX}}= \\
15 \mathrm{~cm}\end{array}$ & $0.005 \mathrm{~m}$ & $\begin{array}{l}0.01 \\
\mathrm{~m}\end{array}$ & $\begin{array}{l}0.015 \\
\mathrm{~m}\end{array}$ & $0.02 \mathrm{~m}$ & $\begin{array}{l}0.02 \\
\mathrm{~m}\end{array}$ & $\begin{array}{l}0.08 \\
\mathrm{~m}\end{array}$ \\
\hline
\end{tabular}
in the study cases $(\mathrm{d} / \mathrm{i}$ deflated/inflated).

Table III. Propagation time of the reflected signal

\begin{tabular}{|l|l|l|}
\hline $\begin{array}{l}\text { Antenna distance } \\
\mathbf{d}_{\text {A_tot }}\end{array}$ & $6.5 \mathrm{~cm}[2-8 \mathrm{GHz}]$ & $15.0 \mathrm{~cm}[3-10 \mathrm{GHz}]$ \\
Time [s] & & \\
\hline$t_{\mathrm{TX} \_\mathrm{w}}$ & $1.5 \mathrm{e}-10$ & $1.4 \mathrm{e}-10$ \\
\hline$t_{\mathrm{W} \_\mathrm{RX}}$ & $1.2 \mathrm{e}-9$ & $2.4 \mathrm{e}-9$ \\
\hline$t_{\text {total_wire }}$ & $1.3 \mathrm{e}-9$ & $2.5 \mathrm{e}-9$ \\
\hline$t_{\mathrm{TX} \_\mathrm{V}}$ & $1.2 \mathrm{e}-9$ & $1.1 \mathrm{e}-9$ \\
\hline$t_{\mathrm{V} \_\mathrm{RX}}$ & $1.4 \mathrm{e}-9$ & $2.9 \mathrm{e}-9$ \\
\hline$t_{\text {total_valve }}$ & $2.6 \mathrm{e}-9$ & $4.0 \mathrm{e}-9$ \\
\hline
\end{tabular}

The dimensions and relative permittivity of corresponding tissues at different frequencies are obtained from [16] and summarized in Table II. Permittivity values for $2,3,8$, ja 10 $\mathrm{GHz}$ are presented to show the variation of the permittivity values within the studied frequency ranges. When we calculate the velocity in each tissue, we use the permittivity averaged over the frequency range used in the measurements.

\section{MEASUREMENT RESULTS}

\section{A. Antenna distance $6.5 \mathrm{~cm}$}

At first we study the channel impulse responses obtained using the SkyCross antenna in the measurements with the volunteer having an implant. Figure $4 \mathrm{a}$ illustrates all 100 impulse response samples obtained in the measurements when the antenna was on the left side of the body. For comparison, the impulse responses with the reference volunteer (non-implant) 
are shown in Fig. 4b. The variation between the samples are due to the small unintentional movements of the volunteer during the measurement. For both cases, the main peak arrives at same time, approximately $0.5 \mathrm{~ns}$. This is the line-of-sight propagation peak. At time instant $1 \mathrm{~ns}$ arrives the second peak for both cases, assumed the signal propagation through creeping waves.

A rough comparison between the impulse responses presented in Fig 4a and Fig 4b show that there are more peaks at significant signal level in the channel impulse responses of the volunteer with an aortic valve implant. According to the calculations presented in Table III, the peaks due to the metallic wires in the sternum should arrive at the time of approximately $1.3 \mathrm{~ns}$. Some variation is seen in the timing and strength of the peaks because there are several wires and also breathing has impact on this. In Figure $4 a$, the peaks due to the metallic wires can clearly be seen around $1.3 \mathrm{~ns}$. Instead, in the channel IR of the reference person, there is no clear peak at this time set.

According to Table III, the peaks due to the aortic valve should be seen at the time instant of 2.6 ns. With SkyCross antenna, the implanted volunteer has a peak around $2.3 \mathrm{~ns}$ in the measurement, when the RX-antenna is on the left side and 2.5 $\mathrm{ns}$ in the measurements, when the RX-antenna is on the right side. Since the valve is located slightly on the left side of the body center, the reflected signal has to propagate longer distance within the tissues on the right-side measurements, which explains the difference between the timing and attenuation differences in left and right side cases. The level of the peak is relatively low compared to the main peak since the attenuation of the signal within the body tissues is high. Since the valve is moving with the heartbeat, also the reflection angle is changing periodically and thus the arrival timing is changing. This explains the differences between timing of the samples.

Next we study the impulse responses obtained in the measurements with the loop antenna on the volunteer with an aortic valve implant. Both left side and right side cases were studied and the results were shown in Figure 6a and 6b. Similar tendencies can be found as with SkyCross antenna: Peaks due to the metallic wires in the sternum can be found approximately at the time instant of $1.2 \mathrm{~ns}$. Furthermore, the peaks due to the aortic valve implant at the time instant of $2 \mathrm{~ns}$.

\section{B. Antenna distance $15 \mathrm{~cm}$}

Next, we study the measurement results for left-side case where the distance between the antennas is $15 \mathrm{~cm}$. The impulse responses for all 100 samples are plotted for loop and dipole antennas in implant and non-implant cases in Figures $7 \mathrm{a}$ and $7 \mathrm{~b}$, respectively. Additionally, the average of the impulse responses are included in the figures.

According to the calculations presented in Table III, the signal paths due to the reflection from the metallic wires should arrive approximately at the time instant $2.6 \mathrm{~ns}$. From the Figure $7 \mathrm{a}$, we can see clear peaks arriving at this time instant. For some IR samples, the peaks around 2-2.6 ns are clearly higher. However, the average curve shows only a small peak at $2.6 \mathrm{~ns}$ since the arriving peaks are not aligned, which may affect destructively on the peaks of the averaged IR.
Finally, the reflections from the aortic valve are evaluated. Table III shows that the signal paths reflected from the valve should arrive approximately at time instant $4.0 \mathrm{~ns}$. When looking at the averaged impulse response (black dashed line) in Figure 8a, there is no peak visible in the impulse response. Instead, when we study the single impulse responses more precisely, we can notice very low peaks in some of the IR samples. This is due to the fact that aortic valve is moving and hence causing variations in the reflections. Due to the strong attenuation of the signal within the tissues, the level of the peak is very low as the antenna distance is $15 \mathrm{~cm}$. When studying the averaged channel IR (blue solid line) of the reference volunteer, we see also several peaks but those at the considered time instant are almost at noise level. These results are valid both for loop and dipole antennas.

\section{SUMMARYAND DISCUSSION}

In this paper, we have examined the impact of the sternotomy wires and aortic valve implant on the UWB channel characteristics. The evaluations are performed by calculations, measurement data analysis, and FIT-based power flow simulations. In the measurements, we used three different antennas designed for on-body and off-body communications.

From the results it was found that the sternotomy wires and the aortic valve implant have an impact on the channel characteristics. The impact depends on the nature of the antennas and their location. Even with the on-body antennas, the impact can clearly be seen in the measurement results: there are peaks at time instances that correspond to the expected propagation delay from the sternotomy wires and the aortic valve implant. In the calculation of the expected propagation delay, the propagation velocities in different tissues on the propagation path has been taken into account. Naturally, the individual differences may cause strong variation in the results. Furthermore, the impact is also assumed to be clearly more significant if in-body antennas were used instead of on-body antennas.

Previously the impact of the sternotomy wires has not been investigated and, hence, the results presented in this paper provide a first insight to this aspect. The medical wires can be considered as a type of medical implant since they are nonbiological material and normally stay in the body after the operation for the rest of patient's life. Many of them are close to the skin and hence can have a significant impact on the channel characteristics. This has to be taken into account if using any inbody or on-body communication devices, for instance endoscopy capsules, etc., since additional peaks in the channel impulse response may cause interference. Besides, knowledge about the impact of the wires would allow to better locate $\mathrm{Rx}$ and Tx antennas for communications with in-body implants avoiding that interference.

\section{ACKNOWLEDGMENT}

We would like to thank all the volunteers who participated in the experimental measurements. We would also like to thank Dr. Richard Kovacs, Clinical Director of the Krannert Institute of Cardiology, at Indiana University, USA, for his help in gathering technical information about the nature of sternotomy wires and Medtronic aortic valve, and to the Medtronic engineers, who 
participated in the process of locating this information. We also like to thank our internal Wireless In-body Communications (WiB) project for funding.
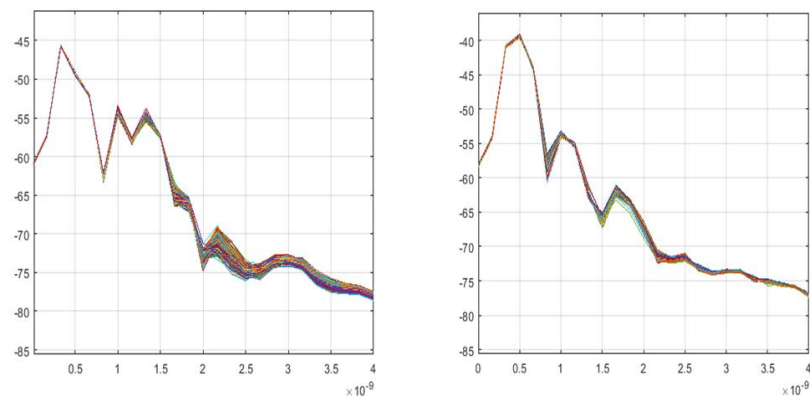

Figure 4. SkyCross antenna IRs a) with implant, and b) without implant in the left side case
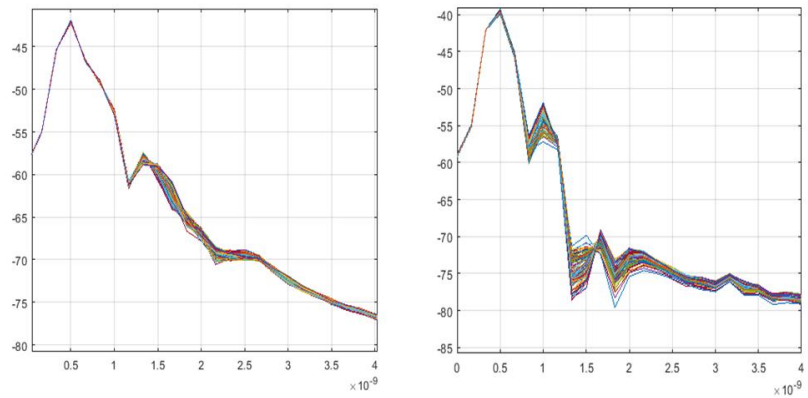

Figure 5. SkyCross antenna IRs a) with implant, and b) without implant in the right side case.
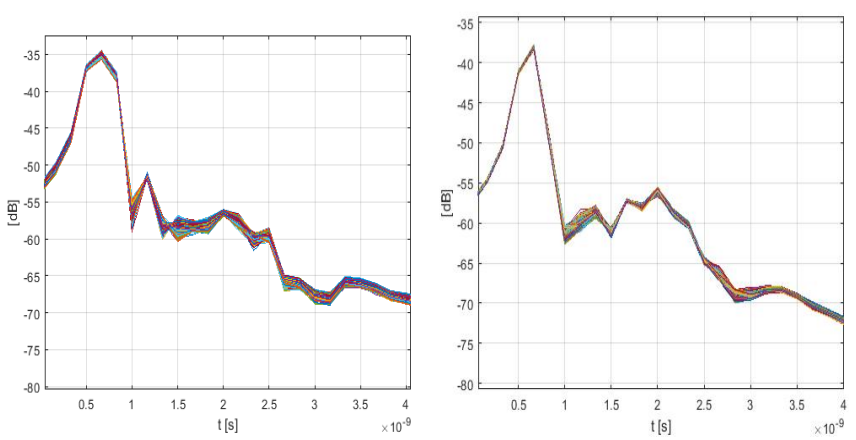

Figure 6. Loop antenna IRs a) at left side measurements and b) right hand measurements
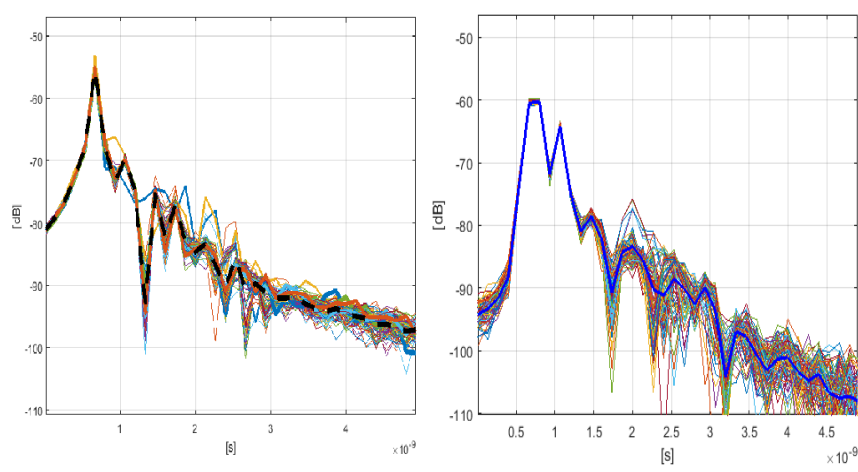

Figure 7. Loop antenna IRs a) with the implant and b) without the implant.
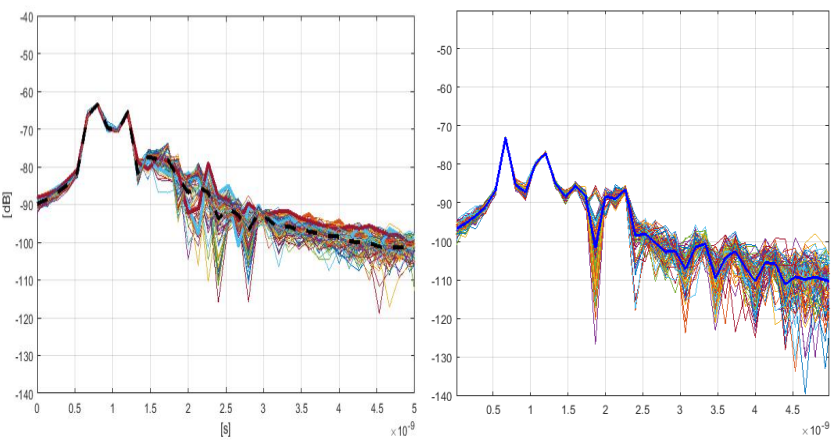

Figure 8. Dipole antenna IRs a) with the implant and b) without the implant.

\section{REFERENCES}

[1] S. Cotton, R. D’Errico, C. Oestges, “A Review of Radio Channel Models for Body Centric Communications," Radio Sci, Jun, 2014. doi: $10.1002 / 2013 R S 005319$.

[2] X. Yang, M. Fang, A. Ren, Z. Zhang, Q. Abbasi, A. Alomainy,K. Mehran, "Reverse Recognition of Body Postures using On-Body Radio Channel Characteristics,"IET Microwaves, Ant., and Prog, 2017.

[3] I. Dove, "Analysis of Radio Propagation Inside the Human Body for inBody Localization Purposes", University of Twente, Netherland, Aug. 2014.

[4] T. Kumpuniemi, M. Hämäläinen, K. Y. Yazdandoost, J. Iinatti, "Human Body Shadowing Effect on Dynamic UWB On-Body Radio Channels," IEEE Antennas and Wireless Propagation Letters, Vol 16, 2017.

[5] T. Kumpuniemi, M. Hämäläinen, K. Y. Yazdandoost, J. Iinatti, "Categorized UWB On-Body Radio Channel Modeling for WBANs," Progress in Electromagnetic Research B, Vol. 67, 2016.

[6] A. Taparugssanagorn, C. Pomalaza-Ráez, A. Isola, R. Tesi,M. Hämäläinen, J. Iinatti, "Preliminary UWB Channel Study for Wireless Body Area Networks in Medical Applications" in International Journal of Ultra Wideband Communications and Systems, InderScience Publishers, vol. 2, no. 1, pp. 14-22, 2011

[7] A. Taparugssanagorn, C. Pomalaza-Ráez, A. Isola, R. Tesi,M. Hämäläinen, J. Iinatti, ”UWB Channel Modeling for Wireless Body Area Networks in Medical Applications," Applied Sciences in Biomedical and Communication Technologies (ISABEL), 2009.

[8] W.-B. Yang, K. Sayrafian-Pour, J. Hagedorn, J. Terrill, K.Y. Yazdandoost, A. Taparugssanagorn, M. Hämäläinen, J. Iinatti, "Impact of an Aortic Valve Implant on Body Surface UWB Propagation: A Preliminary Study", Proc. the 5th International Symposium on Medical Information and Communication Technology, 2011.

[9] M. Särestöniemi, M. Hämäläinen, J. Iinatti, "An Overview of Electromagnetic Propagation Based Channel Modeling Techniques for Wireless Body Area Network Applications," IEEE Access, 2017.

[10] A. Pellegrini et al, "Antennas and Propagation for Body-Centric Wireless Communications at mm-wave Frequencies: A Review,"IEEE Antennas Propagation Mag, Vol. 55, 2013.

[11] A.Elfström, A. Grunditz, "Evaluation of Sternum Closure Techniques Using Finite Element Analysis," Master Thesis, in Medical Engineering, The Royal Institute of Technology, Sweden, 2013.

[12] T. Tuovinen, K. Yekeh Yazdandoost, J. Iinatti, "Comparison of the Performance of Two Different UWB Antennas for the use in WBAN OnBody Coimmunications," $6^{\text {th }}$ Europ. Conf. Antennas and Propagation (EUCAP), 2012.

[13] SkyCross Antenna Product, www.skycross.com

[14] CST Microwave Studio, [Online]. Available: http://www.cst.com.

[15] Medtronic aortic valve, URL:http://www.15tronic.com/us-en/healthcareprofessionals/products/cardiovascular/heart-valves-surgical.html

[16] https://www.itis.ethz.ch/virtual-population/tissueproperties/database/dielectric-prope 